\title{
La sécurité des grandes villes face au risque de contamination des eaux de consommation
}

Approche théorique de la régulation des flux hydriques au sein du territoire urbain

Big cities security facing risk of drinking water pollution : theoretical approach to the regulation of groundwater flows within the city

Die Sicherheit der Grosstädte im Hinblick auf die Verunreinigung der

Trinkwässer gegenüber : theoretische Skizze von wässerig Regulierungsflut im

Stadtgebiet

\section{Claudine Ferron}

\section{(2) OpenEdition}

\section{Journals}

Édition électronique

URL : http://journals.openedition.org/rge/4453

DOI : $10.4000 /$ rge.4453

ISSN : 2108-6478

Éditeur

Association des géographes de l'Est

Édition imprimée

Date de publication : 1 avril 1999

ISSN : 0035-3213

Référence électronique

Claudine Ferron, "La sécurité des grandes villes face au risque de contamination des eaux de consommation », Revue Géographique de l'Est [En ligne], vol. 39 / 2-3 | 1999, mis en ligne le 05 septembre 2013, consulté le 08 septembre 2020. URL : http://journals.openedition.org/rge/4453 ; DOI : https://doi.org/10.4000/rge.4453

Ce document a été généré automatiquement le 8 septembre 2020 .

Tous droits réservés 


\title{
La sécurité des grandes villes face au risque de contamination des eaux de consommation
}

\author{
Approche théorique de la régulation des flux hydriques au sein du \\ territoire urbain \\ Big cities security facing risk of drinking water pollution : theoretical approach \\ to the regulation of groundwater flows within the city \\ Die Sicherheit der Grosstädte im Hinblick auf die Verunreinigung der \\ Trinkwässer gegenüber : theoretische Skizze von wässerig Regulierungsflut im \\ Stadtgebiet
}

\section{Claudine Ferron}

1 La gestion de la pollution accidentelle des eaux destinées à la consommation humaine en ville revêt deux aspects indissociables : le premier est lié aux phénomènes générant la situation de crise induite par le déversement intempestif de matières dangereuses à proximité des puits de captage alimentant en eau la population urbaine, le second provient de l'organisation des autorités, dont le rôle est de prévenir et de gérer ces événements.

2 Si on connaît assez bien les mécanismes élémentaires responsables de la contamination des eaux distribuées en ville, ainsi que les modes de régulation mis en place par les pouvoirs publics pour en limiter les effets, le problème reste de comprendre comment ces deux phénomènes interagissent entre eux. Nous traiterons des représentations à partir desquelles nous avons pu élaborer des propositions méthodologiques pour connaître la nature et l'intensité de ces interactions. 


\title{
I. La pollution accidentelle des eaux destinées à la consommation humaine en ville
}

\author{
A. Constat : la rémanence du risque de pollution des eaux de \\ consommation en ville en dépit d'un haut niveau de sécurité
}

3 Les dangers que peuvent faire courir aux populations urbaines une pollution accidentelle des eaux de consommation ont conduit à porter à un très haut niveau de sécurité le dispositif technique de distribution d'eau potable. Pour autant, ce haut niveau de sécurité ne signifie pas que le risque de contamination des eaux distribuées ait été définitivement éliminé. Malgré l'existence de moyens de surveillance et d'intervention de plus en plus élaborés, on observe la persistance de cas de pollution des eaux destinées à la consommation urbaine.

4 Cet état de fait est au centre des paradoxes propres aux systèmes technologiques à risques auxquels le dispositif d'approvisionnement en eau des villes appartient. D'un côté, ces dispositifs s'avèrent extrêmement performants : un très haut niveau de fiabilité est obtenu dans le transport de l'eau en ville, au travers de l'application de techniques de plus en plus complexes de sécurité récurrente. D'un autre côté, les risques pesant sur ce dispositif d'approvisionnement, telles les pollutions accidentelles des captages, sont devenus plus graves et plus intolérables qu'auparavant : le nombre de pannes de distribution d'eau potable augmente - citons tout particulièrement les cas de Lyon et de Tours, toutes deux sérieusement affectées par le déversement accidentel de matières dangereuses menaçant la desserte en eau de l'ensemble de la population citadine -, créant des situations de plus en plus difficilement vécues par les collectivités.

5 Cette situation paradoxale nous conduit à poser la question de la sécurité d'approvisionnement en eau potable sous deux formes : doit-on admettre qu'il existe une limite de sécurité dans ces grands systèmes que sont les dispositifs techniques de distribution d'eau potable en ville? Si l'on admet que l'on peut encore progresser, comment cette limite peutelle être franchie?

6 Ces deux questions entraînent des discussions de nature différente. La première, l'idée d'une limite irrémédiable en matière de sécurité, conduit à proposer un modèle susceptible d'expliquer cette limite et à trouver un moyen de rationaliser et d'accepter ce constat. La seconde conduit à examiner les solutions actuelles, à les critiquer et si possible, à proposer de nouvelles pistes. C'est cette seconde approche que nous avons choisie.

\section{B. Remise en question du dispositif actuel de lutte : la domestication du risque et ses limites}

7 La dynamique de transfert d'un polluant à proximité d'un puits de captage est un phénomène complexe. Cette complexité tient à la plus ou moins grande prédictibilité du phénomène et à son irréversibilité.

Le manque de prédictibilité est un important facteur de complexité. Le lieu et le moment du déversement du polluant à la surface du sol sont difficilement détectables et la dynamique de propagation du flux au sein de la nappe, bien que prévisible à l'aide 
de modèles, est sujette à des fluctuations dues aux nombreux facteurs qui la conditionnent. Avec la quantité de plus en plus élevée de matières dangereuses utilisées dans l'industrie, les lieux probables de déversement accidentel à proximité des puits de captage augmentent. Le caractère peu prédictible du risque accidentel de contamination des eaux distribuées en ville entraîne une limitation des anticipations et une conduite à court terme.

9 La réversibilité décrit la possibilité pour les autorités chargées de maîtriser le risque de pollution d'annuler une action par une action inverse. Or, il n'est jamais possible d'obtenir une réversibilité totale au niveau du contrôle de la contamination des eaux destinées à la consommation humaine du fait de l'évolution de ce processus. Lorsque le puits de captage est atteint, le flux de polluant s'introduit dans le réseau public de distribution et menace la sécurité d'approvisionnement en eau potable sur l'ensemble des lieux desservis. Dès lors, les seules actions possibles consistent à limiter les conséquences des problèmes, sans jamais pouvoir revenir à la situation initiale.

De telles caractéristiques, spécifiques au risque accidentel de pollution, permettent d'expliquer certaines limites fondamentales d'une approche exclusivement technicienne de la sécurité d'approvisionnement en eau des grandes villes. La complexité du dispositif assurant l'alimentation en eau potable d'une agglomération rend en effet difficile une approche prenant comme seul critère la probabilité d'apparition du risque et la gravité des conséquences d'un accident potentiel.

\section{Vers une nouvelle approche de gestion du risque de pollution des eaux de consommation en ville}

11 L'irréductibilité du risque de pollution accidentelle et l'incertitude qui lui est rattachée actualisent une problématique fondée sur son acceptation et sa gestion. Compte tenu de l'imbrication des différents composants du dispositif technique de distribution, un risque pesant sur un de ses éléments est susceptible d'engendrer des conséquences sur l'ensemble de la chaîne d'alimentation, d'où une difficulté certaine à apprécier l'impact des différents risques et à évaluer l'apport des mesures correctives à appliquer.

12 Selon cette optique, la sécurité d'approvisionnement en eau, à l'échelle d'une agglomération donnée, requiert un développement des opérations de surveillance et d'intervention qui ne peuvent se limiter aux seuls points névralgiques du dispositif de distribution mais doivent, au contraire, s'étendre à l'ensemble du réseau ainsi qu'à son environnement physique et social.

13 Ce modèle, s'il est vérifié, tendrait alors à remettre en question les formes d'organisation sociale qu'implique la régulation actuelle des flux hydriques en milieu urbain. Il ne s'agit plus d'abstraire le risque de son contexte et d'appliquer des mesures normatives censées favoriser son élimination. Le choix des mesures de lutte doit désormais être orienté vers sa maîtrise, au travers de la saisie des dynamiques qui structurent chaque situation particulière. 


\section{L'eau en ville : un hydro-socio-système}

\section{A. La sécurité d'approvisionnement en ville : le produit d'une organisation} versant, soit par des limites administratives tels une région, un département ou une commune, est le champ spatio-temporel d'une partie du cycle général de l'eau dans l'hydrosphère. Un tel champ constitue un système nommé hydro-socio-système. Nous entendons par système « un ensemble d'éléments en interactions dynamiques, organisé en fonction d'un but » (De Rosnay, 1975).

Un hydro-socio-système urbain est constitué de plusieurs entités spatio-temporelles au niveau desquelles circulent des flux de nature et d'intensité distincts. Ces entités sont d'une part, le réseau physique d'écoulement souterrain et de surface, caractérisé par une structure hydrogéologique et hydrologique plus ou moins complexe et d'autre part, le réseau technique de distribution d'eau potable, à structure ramifiée. Ces deux entités constituent elles-mêmes des systèmes. Premièrement, l'eau fait partie du milieu physique dans lequel la ville se développe. Ses caractéristiques sont celles d'un système naturel, composé d'organismes vivants entre lesquels circulent des flux de matière et d'énergie, au sein de plusieurs milieux en interaction, tels les fleuves, les rivières, les nappes souterraines et les surfaces en eau. Deuxièmement, l'eau fait partie de l'édifice urbain lui-même. Ses caractéristiques sont aussi celles d'un système social, composé d'individus en relation, tels les « usagers ", «les gestionnaires » et les "décideurs", 
entre lesquels circulent des flux de matière et d'information de nature et d'intensité diverses.

\section{Principaux constituants} eau potable d'une collectivité urbaine, maîtrisant ses effluents et luttant contre le risque de pollution de ses puits de captage, nous avons considéré quatre types de soussystèmes :

- le sous-système physique de circulation de l'eau, composé de deux catégories principales d'éléments, le réseau hydrographique superficiel et le réseau souterrain, reliés entre eux par des flux; des acteurs socio-économiques, simples usagers et filières de gestion, organisés en fonction d'un but, l'utilisation de l'eau destinée à la consommation humaine;

- le sous-système technique de circulation de l'eau, formé de l'ensemble des ouvrages techniques, stations de pompage, réservoirs et station d'épuration. Ces ouvrages assurent la distribution et la collecte des eaux potables et usées auprès des usagers raccordés aux réseaux d'alimentation et d'assainissement. Leur fonction est de traiter les flux d'eau reçus et émis par chaque élément constitutif du système socioéconomique d'utilisation de l'eau ;

le sous-système juridico-institutionnel de protection de la ressource en eau potable, ensemble de règles définissant les caractéristiques physico-chimiques de l'eau potable et le mode d'occupation du sol à proximité des captages au sein d'un espace déterminé, les périmètres de protection. À cet espace est associé un dispositif de contrôle et d'intervention en cas de dépassement de seuil dû à l'apparition, en un lieu à un moment donné, d'une pollution accidentelle.

Ces quatre sous-systèmes participent à la pérennité et à la stabilité de l'organisation globale qu'est l'hydro-socio-système à l'échelle d'une agglomération urbaine, chacun d'eux étant dépendant des autres : la mobilisation de la ressource en eau, extraite du système physique de circulation, est effectuée par le système technique de distribution d'eau potable, à la demande du système socio-économique d'utilisation et sous le contrôle du système juridico-institutionnel de surveillance et de contrôle de la qualité de l'eau distribuée.

\section{Propriétés spatiales et temporelles}

Un hydro-socio-système urbain présente une dynamique spatio-temporelle spécifique : l'eau circule au sein d'un territoire donné, selon une trajectoire particulière, celle que lui imprime son cours naturel, à laquelle s'ajoute celle que lui donne le réseau technique de distribution. Son parcours au travers du tissu urbain s'effectue suivant une cynétique déterminée soit par des processus physiques - la vitesse d'écoulement des eaux souterraines au sein de l'aquifère par exemple -, soit par des processus techniques - le débit de pompage des captages ou la pression au sein des canalisations.

La première dimension à prendre en considération pour analyser la structure et le fonctionnement de l'hydro-socio-système urbain est sa dimension spatiale. Il est

Revue Géographique de l'Est, vol. 39 / 2-3 | 1999 
difficile de déterminer les limites au-delà desquelles les relations s'établissant entre les éléments constitutifs du système sont moins fortes que celles établies avec le milieu extérieur. Les raisons en sont les suivantes :

les réseaux de distribution d'eau potable sont conçus pour desservir une aire qui s'étend bien au-delà du territoire d'une agglomération. En effet, le territoire couvert par ces réseaux concerne le plus souvent plusieurs communes, regroupées en un syndicat intercommunal. D'autre part, même lorsqu'une agglomération est alimentée par un seul réseau, dont les limites sont celles de son territoire, ce dernier est interconnecté avec les réseaux de distribution des communes voisines ;

- la rivière ou la nappe qui alimente en eau l'agglomération urbaine s'étend bien audelà du territoire urbain, son écoulement s'effectuant au sein d'un bassin-versant ou d'un aquifère plus ou moins vaste ;

- les points de prélèvement desservant en eau la ville sont théoriquement situés hors agglomération, loin des habitations et des zones d'activités susceptibles de nuire à la qualité des eaux ;

conformément à la loi sur l'eau de 1992, la gestion de la ressource, notamment des rivières, s'effectue de plus en plus au sein de grandes entités de gestion, les bassinsversants, au niveau desquels des règles d'utilisation sont élaborées en concertation avec l'ensemble des acteurs, usagers, gestionnaires et autorités administrative, localement implantés au sein de ces bassins.

Pour toutes ces raisons, il nous est apparu que l'espace à prendre en compte correspond à l'aire d'alimentation des captages approvisionnant en eau les villes, appelée également champ captant. Cet espace ainsi que le dispositif juridicoinstitutionnel de contrôle et de surveillance de la qualité des eaux qui y est associé, constituent un "modèle réduit " illustrant la complexité des rapports s'établissant entre les processus physiques participant au transfert de polluants dans les eaux destinées à la consommation humaine d'une part, et les processus décisionnels relatifs à la protection de la ressource d'autre part.

33 La seconde dimension à prendre en considération est la dimension temporelle. La distinction entre le processus de propagation du flux de pollution et le processus de réaction de l'organisation conduit à séparer la dynamique de transfert du polluant et la pression temporelle associée au processus de décision des autorités selon deux temporalités :

- la temporalité propre au processus de propagation du polluant se définit par la durée moyenne des niveaux de concentration de l'eau en différentes substances en un point et à un moment donnés et par la vitesse de transition d'un niveau à un autre. Ce temps s'impose aux différentes autorités chargées d'intervenir en cas de pollution accidentelle des puits de captage. Il correspond à la durée dont disposent les autorités administratives pour prendre leur décision et agir sur les sous-systèmes physique, technique et/ou socio-économique ;

le temps de réaction de l'organisation est le produit de l'expérience acquise en matière de lutte contre la pollution, permettant au gestionnaire de la distribution d'eau potable d'une ville et à l'ensemble des services intervenant en cas de contamination des eaux distribuées de s'adapter de façon telle que leur comportement s'ajuste aux conséquences temporelles de l'environnement. 


\section{Les mécanismes à étudier au sein de l'hydro-socio- système urbain} institutionnel de lutte contre la pollution accidentelle des puits de captage passe par l'examen de la nature des mécanismes d'ajustement établis au sein de l'hydro-sociosystème urbain et de leurs limites. L'examen de ces mécanismes nécessite que soient étudiées trois propriétés propres à tout système complexe : son équilibre, sa plus ou moins grande stabilité et sa capacité d'adaptation.

\section{A. L'équilibre du système}

L'équilibre de l'hydro-socio-système urbain est déterminé par l'intensité des échanges s'établissant entre le système physique de circulation et le système socio-économique d'utilisation. Le système physique de circulation se définit comme un système d'offre, réunissant des potentialités biologiques, écologiques, techniques et récréatives. Le système socio-économique d'utilisation peut être considéré comme un système de demande correspondant aux besoins et aux aspirations des hommes et des activités sur le territoire urbain. L'équilibre s'établit lorsque l'offre disponible, soit l'eau prélevée, correspond à la demande d'approvisionnement, relative aux volumes d'eau considérés comme nécessaires pour répondre aux besoins des usagers. Cette mise en correspondance s'obtient par le moyen d'actions visant à corriger les écarts possibles entre les flux extraits du système physique et les demandes provenant du système socio-économique. Une partie du flux naturel est transformé en stock dans un ou plusieurs réservoirs. Suivant le niveau de ces derniers, les volumes d'eau distribuée équilibrent les volumes d'eau prélevée, ce qui permet au gestionnaire de la ressource de pourvoir, en tout lieu et à tout moment, aux besoins des usagers implantés sur le territoire urbain. Une "autonomie» d'un nombre d'heures déterminées est ainsi assurée, compte tenu des réserves constitutives de l'hydro-socio-système.

\section{B. L'instabilité du système}

L'instabilité de l'hydro-socio-système urbain est importante. Elle est due à la confrontation de deux logiques différentes dont son fonctionnement dépend : celle relevant du système physique et celle relevant du système social.

En tant que sous-système de l'hydrosphère, le système physique de circulation de l'eau en milieu urbain se caractérise par l'existence d'un équilibre dynamique entre les flux entrants, les flux sortants et les variations de stocks et par une aptitude à répondre aux impulsions extérieures. Des mécanismes complexes assurent la stabilité du système par une articulation précise des stocks avec les flux, ce qui lui permet de revenir à son état initial après avoir été perturbé.

40 L'activité socio-économique propre à la distribution d'eau potable en milieu urbain relève d'une logique tout à fait différente. En vue d'assurer la sécurité d'approvisionnement de la population citadine, le service des eaux d'une ville, comme toute organisation économique, transforme des moyens de production, l'eau prélevée, en produits fabriqués. Cette transformation répond à un caractère d'efficience : les flux d'eau issus du système physique sont gérés en fonction des niveaux de consommation

Revue Géographique de l'Est, vol. 39 / 2-3 | 1999 
et des objectifs de production des hommes et des activités implantés sur le territoire urbain.

41 L'augmentation de la population et l'intensification des échanges en milieu urbain constituent une menace pour l'équilibre de l'hydro-socio-système. Lorsque l'activité du système urbain s'accélère, les relations que la ville établit avec le système physique de circulation s'intensifient. Si les prélèvements d'eau deviennent trop importants, le déficit des ressources locales n'est plus compensé par les précipitations efficaces et l'exploitation des captages se fait au détriment des réserves. De même, l'apport de substances polluantes dû à la densification du tissu urbain à proximité des captages peut être à l'origine d'une rupture d'équilibre, plus ou moins durable, du système physique de circulation.

\section{Les capacités d'adaptation du système}

42 Les capacités d'adaptation de l'hydro-socio-système urbain aux perturbations extérieures sont plus ou moins importantes. La constitution de réserves, comme il a été dit précédemment, constitue un premier degré dans la production d'autonomie au sens où l'entend P. Vendryes (1989): «en acquérant son autonomie, à partir du milieu extérieur et par rapport à lui, l'organisme acquiert la possibilité d'entrer avec lui en relation aléatoire ». Vis-à-vis de perturbations et d'aléas issus de son environnement, l'hydro-socio-système urbain ne se limite pas à des actions défensives fondées sur la mobilisation de réserves physiques. Un deuxième degré dans la production d'autonomie est atteint, qui correspond à la constitution et à l'exploitation de réserves cognitives (informations sur les flux). Des connaissances sont produites à partir d'informations sur les caractéristiques physico-chimiques de l'eau, acquises en différents points de l'hydro-socio-système, et l'état de qualité que celle-ci doit présenter pour satisfaire les besoins des hommes et des activités en ville. Ces connaissances conditionnent la mise en place d'un dispositif de correction assurant le maintien de cet état de qualité en dépit des aléas.

\section{Principes de modélisation}

\section{A. Principal élément à prendre en compte : les flux d'information}

43 L'information est la caractéristique essentielle à prendre en compte pour examiner la nature et les limites des mécanismes d'ajustement opérés par l'hydro-socio-système urbain lorsque surviennent des modifications de son fonctionnement dues à des perturbations extérieures.

Cette affirmation s'appuie sur les théories de l'organisation biologique et sociale qui considèrent les processus d'émergence de systèmes auto-organisés à partir des processus d'acquisition, de production et de transmission d'informations à des fins d'adaptation (Ashby, 1962).

Pour développer son autonomie, une entité doit avoir une bonne compréhension d'ellemême et de ce qui l'entoure. Dépendante pour partie de son environnement, une entité autonome développe des capacités de cognition lui permettant de générer de façon endogène des informations, d'interpréter ce qu'elle perçoit et de développer des stratégies d'adaptation. Elle peut alors transformer son comportement en fonction des 
perturbations perçues au sein de cet environnement et de ce qui lui est possible de faire, tout en conservant son identité.

\section{B. La démarche de formalisation développée par De Latil}

La compréhension du fonctionnement et de la dynamique de l'hydro-socio-système urbain nécessite l'utilisation d'un outil de recherche construit pour simuler un ensemble de processus en interaction. Parmi l'ensemble des moyens d'investigation systémique, nous avons choisi d'utiliser la cybernétique. La théorie cybernétique est fondée sur la rétroaction et l'information. Un modèle général de la dynamique de fonctionnement de l'hydro-socio-système urbain est proposé sur la base de cette théorie et en particulier du concept d'effecteur développé par De Latil (1953).

Les notions sur lesquelles cette approche est fondée sont les suivantes : un effecteur est un mécanisme produisant un effet. L'effet utile d'un effecteur est celui qui accomplit la finalité de cet effecteur. Un facteur est un élément nécessaire au fonctionnement de l'effecteur. Conduire un effecteur, c'est lui donner un but, qui peut être variable et c'est régler les valeurs des facteurs pour que le but soit atteint ou approché. Le réglage peut se faire soit par interaction d'un facteur (réglage par interaction) soit par rétroaction d'un effet (réglage par rétroaction). Dans son principe, la rétroaction est une fonction reliant un effet à sa cause; pratiquement, c'est une chaîne d'effecteurs, dont l'un joue un rôle de détecteur, et l'autre de correcteur.

À partir de cette approche, il est possible d'expliquer les processus physiques mis en jeu dans la propagation du front de pollution, les dysfonctionnements du dispositif technique de distribution d'eau potable ainsi que les activités de régulation opérées par les différentes autorités de gestion en cas de crise.

\section{Application : la sécurité d'approvisionnement en eau potable de la communauté urbaine de Strasbourg}

49 L'application des concepts et méthodes, énoncés précédemment, au niveau de la Communauté Urbaine de Strasbourg (CUS), permet d'éprouver et d'affiner la démarche.

\section{A. La nappe phréatique rhénane et la CUS}

50 La nappe souterraine, à la hauteur de Strasbourg, a une largeur de plus de $20 \mathrm{~km}$. Son épaisseur croît d'Ouest en Est, passant de $50 \mathrm{~m}$ près de Holtzheim à $100 \mathrm{~m}$ au niveau du Polygone. Son toit se situe à des niveaux très variables : au nord de Strasbourg, il apparaît en surface, alors que dans le secteur d'Oberschaeffolsheim, la nappe est profonde (5-7 m). Son écoulement se fait globalement du Sud vers le Nord, à une vitesse de quelques mètres par jour.

51 Cette réserve d'eau souterraine offre à la CUS de nombreux avantages. Le volume d'eau stockée assure à la communauté urbaine une richesse quasi inépuisable en eau. Celle-ci, qualifiée d'eau bicarbonatée calcique, permet la plupart des usages. En outre, la nappe est facilement accessible; elle est atteinte par des puits creusés à faible profondeur, de quelques mètres à quelques dizaines de mètres au plus et cela sur le lieu même de la demande. 

d'étude est justifié par le fait que ce secteur est fortement exposé au risque de pollution: le recensement des risques effectué par la DRIRE révèle en effet près de cinquante sources potentielles de pollution. Parmi celles-ci, citons d'importants dépôts d'hydrocarbures stockés au niveau du Port-aux-Pétroles, les pipelines transportant le pétrole brut et les produits dérivés, ainsi que les conduites maîtresses du réseau d'eaux usées de l'agglomération strasbourgeoise. En cas de déversement accidentel, la propagation de matières dangereuses au sein de l'aquifère, suivant le sens d'écoulement général des eaux qui est Sud-Ouest/ Nord-Est, expose tout particulièrement le puits de la Robertsau au risque de pollution.

\section{B. Structure et fonctionnement de l'hydro-socio-système}

Conformément aux principes d'analyse énoncés précédemment, l'étude de l'hydrosocio-système urbain dans lequel s'inscrit le puits de captage d'eau potable de la Robertsau comporte trois sous-parties : définition, délimitation et partition du système.

Rappelons la définition que nous avons donnée d'un hydro-socio-système urbain : la sécurité d'approvisionnement en eau potable de la population à l'échelle d'une agglomération est, à la fois, l'objet et le produit d'une organisation, nommée hydrosocio-système. Le système dont il est ici question correspond donc à l'ensemble des éléments physiques et sociaux participant à la distribution d'eau potable auprès de la population desservie par le puits de la Robertsau et implantée sur le territoire de la CUS.

Cet hydro-socio-système possède une frontière à l'intérieur de laquelle les interactions entre les éléments le constituant sont plus fortes que celles établies avec l'extérieur. Étant donné l'attention que nous portons à l'exposition du puits de la Robertsau au risque de pollution, nous retenons comme limite du système la zone d'appel du captage.

$\mathrm{Au}$ sein de cette zone, tous les éléments constitutifs de l'hydro-socio-système, tant physiques que sociaux, participent directement ou indirectement à la sécurité d'approvisionnement en eau potable de la population desservie par le puits de la Robertsau. Ces éléments sont en interaction dynamique : les échanges qu'ils établissent entre eux déterminent la qualité de l'eau pompée dans cette zone. Ils forment donc un système, élément d'un système plus vaste, l'hydro-socio-système propre au territoire de la CUS tout entier.

\section{Application du formalisme De Latil}

L'hydro-socio-système dans lequel s'inscrit le puits de la Robertsau s'apparente à un effecteur, dont l'analyse des éléments constitutifs est décrite ci-après.

Dans le secteur étudié, le dispositif physique de circulation comprend plusieurs cours et plans d'eau reliés à la nappe phréatique rhénane (facteur). Le sens général d'écoulement des lignes de courant des eaux souterraines est S-SW/N-NE. Les cours d'eau en relation avec la nappe phréatique rhénane sont le Rhin canalisé, l'ill et le Steingiessen. À ces trois cours d'eau sont reliés deux canaux : le canal de la Marne-auRhin et le canal de la Papeterie. L'ensemble des plans d'eau situés à l'intérieur de la zone d'appel du puits de la Robertsau sont au nombre de deux : la gravière Vogelau à Schiltigheim et le bassin A. Auberger.

Revue Géographique de l'Est, vol. 39 / 2-3 | 1999 
59 La CUS dispose de son propre service de distribution d'eau potable (effecteur). Ce service est une régie sans personnalité morale ni autonomie financière. Elle ne comporte pas d'organes spécialisés de gestion, les décisions la concernant étant prises par le conseil de la communauté. Le dispositif d'approvisionnement en eau est composé d'organes et d'un centre de commande liés entre eux par des flux d'information en vue d'assurer l'extraction, l'adduction, le stockage et la desserte de l'eau en différents lieux du territoire.

60 L'eau est prélevée dans la nappe par quatre stations de pompage, situées respectivement au Polygone, à Obershaubergen, à Lingolsheim et à la Robertsau. Le puits de la Robertsau est situé dans la forêt du Rhin, près de la maison forestière "Unterjaegerhof », au nord du cours d'eau Steingiessen. Décidé au début des années 1960, il fut mis en service en 1963. La création d'une nouvelle station de pompage a été la solution retenue par la Ville de Strasbourg pour améliorer l'alimentation en eau potable du quartier de la Robertsau. En effet, suite au développement de nouveaux quartiers résidentiels au nord de la Robertsau, la pression de l'eau dans le secteur était devenue insuffisante. La station de pompage de la Robertsau est constituée d'un forage, d'un puits collecteur et d'une moto-pompe. Ce forage est creusé à une profondeur de $17,50 \mathrm{~m}$. La quantité d'eau prélevée est de $130 \mathrm{~m} 3 / \mathrm{h}$.

61 L'objectif principal poursuivi par le Service de l'Eau de la CUS est de garantir la sécurité d'approvisionnement en eau potable auprès de la population qu'il dessert (effet). Le nombre d'usagers raccordés au réseau de distribution communautaire est de 377976 pour l'année 1995, ce qui correspond à 44120 abonnés d'après le schéma directeur de distribution en eau potable BRGM-HYDRATEC. Ils se répartissent en deux catégories définies par une consommation inférieure ou supérieure à $6000 \mathrm{~m} 3 / \mathrm{an}$. En 1995, les usagers gros consommateurs d'eau ne représentent que 1,4\% du nombre des abonnés (651 abonnés). Parmi ceux-ci, citons essentiellement les établissements relevant de l'industrie d'extraction de matériaux (sablières), de l'industrie de transformation (métallurgie, sidérurgie, papeterie), de l'industrie chimique et du secteur agroalimentaire (brasserie) particulièrement bien représentés sur le territoire de la CUS. D'autres usagers sont également de gros consommateurs d'eau, comme les établissements industriels, les grandes surfaces commerciales, les hôpitaux, les écoles et les complexes sportifs. Au total, ce nombre réduit de gros abonnés consomme à lui seul le tiers du volume total d'eau distribuée par la CUS.

La principale mesure de protection dont bénéficie le puits de captage de la Robertsau consiste en la mise en place d'un périmètre, conformément à la réglementation en vigueur. Une réflexion est actuellement en cours pour doter le service de l'eau de la CUS d'un dispositif de surveillance et d'alerte en matière de qualité des eaux. Ce réseau comprend actuellement les points de prélèvement de la DDASS et devrait être complété par la mise en place de stations d'analyse automatique permettant la détection par le Service de l'eau d'une pollution accidentelle à proximité des puits de captage.

Pour connaître l'état optimal de qualité que l'eau doit présenter, la DDASS et le Service de l'Eau de la CUS se référent à des indicateurs, issus de directives nationales ou européennes. Ces directives fixent des critères physiques, chimiques, biologiques et bactériologiques auxquels l'eau doit répondre pour permettre les différents usages dont elle fait l'objet. Pour chacun de ces critères, des valeurs seuils sont définies, que les gestionnaires érigent en objectif de qualité. Pour atteindre ces objectifs, des moyens importants sont déployés : des détecteurs de l'état de qualité de l'eau sont mis en place 
sur le lieu même d'utilisation et des procédures d'intervention plus ou moins complexes sont engagées. Lorsque les critères physiques, chimiques, biologiques ou bactériologiques de l'eau sont non conformes aux valeurs de référence, des actions sont exercées par les gestionnaires pour restaurer l'état de qualité de l'eau : les causes à l'origine de l'écart à la norme sont identifiées et des solutions permettant d'atteindre l'objectif de qualité sont recherchées.

\section{Conclusion}

L'approche développée par De Latil présente l'intérêt de permettre l'étude des actions menées par les autorités de gestion au travers de la prise en compte, au sein du processus décisionnel, des comportements de chacune de ces autorités. La modélisation assure l'intégration de différents niveaux d'interaction et l'émergence, au niveau global, de phénomènes non perceptibles au niveau local. En particulier, la délimitation des rôles et des modes d'intervention des instances décisionnelles au sein du dispositif de régulation pour permettre la mise en évidence des champs de compétence antagonistes, ainsi que des cumuls de fonction à l'origine d'écarts préjudiciables entre le temps de réponse des instances et la dynamique de propagation du polluant.

\section{BIBLIOGRAPHIE}

Ashby (1962). - Principles of the self-organizing system. In : Von Foerster H., Principles of selforganisation, Pergamon Press, New-York.

Bertalanfy L. (1993). - Théorie générale des systèmes, Collection Systémique, Éd. Dunod, 308 p.

De Latil P. (1953). - La pensée artificielle. Introduction à la cybernétique, Éd. Gallimard, Coll. L'avenir de la science, $325 \mathrm{p}$.

De Rosnay J. (1975). - Le macroscope. Vers une vison globale, Éd. du Seuil, Coll. Points Essais, Paris, $346 \mathrm{p}$.

Isnard H., Racine J.B., Reymond H. (1981). - Problématiques de la Géographie, Presses Universitaires de France, Paris, 262 p.

Racine J.B., Reymond H. (1973). - L'analyse quantitative en géographie, Presses universitaires de France, Paris, $311 \mathrm{p}$.

Vendryes P. (1989). - Autonomie, aléatoire et libre arbitre. In : Perspectives Systémiques, dir. B. Paulré, Lyon, pp. 17-36.

\section{RÉSUMÉS}

La recherche a pour but d'évaluer en milieu urbain l'adéquation entre les stratégies de préservation de la qualité de l'eau potable mises en place par les acteurs sociaux et l'exposition 
de la ressource aux risques de pollution. Cette évaluation repose sur l'analyse du nombre et de la nature des relations s'établissant entre l'hydrosphère et le dispositif juridico-institutionnel chargé de limiter les atteintes à la qualité des eaux souterraines en ville. Les éléments descriptifs et spatiaux concernant le front de pollution et les dispositifs de surveillance mis en place par les acteurs sociaux ainsi que leur niveau d'interaction ont été modélisés selon l'approche systémique à partir du modèle développé par De Latil.

The research aims to evaluate for the urban centre the adequacy of strategies for preserving the quality of drinking water established by society, and the exposure of the resources to risks of pollution. This evaluation depends on the analysis of the number and nature of established relations between the hydrosphere and the legal-institutional organisation charged with limiting impacts on the quality of underground water in the town. The descripture and spatial elements of the pollution front and the established surveillance system, as well as their level of interaction has to be modelled according to the systemic approach of the model developed by De Latil.

Die Untersuchung hat das Ziel, im städtischen Milieu die Übereinstimmung zwischen den Schutzmassnahmen der öffentlichen Hand zur Erhaltung der Trinkwasserqualität und der Anfälligkeit dieser Ressource für die Risiken der Verschmutzung abzuschätzen. Diese Abschätzung beruht auf der Analyse von Zahl und Natur der Beziehungen zwischen der Hydrosphäre und den rechtlichinstitutionellen Einrichtungen, die beauftragt sind, die Beeinträchtigung der Grundwasserqualität in der Stadt $\mathrm{zu}$ begrenzen. Beschreibung und räumliche Bezüge der "Verschmutzungsfront» und die Überwachungmassnahmen der öffentlichen Hand wie auch ihr Interaktionsniveau werden hier nach dem von De Latil entwickelten systemtheoretischen Modell dargestellt.

\section{INDEX}

Schlüsselwörter : Sicherheit der Wasserversorgung, Stadt, Systemdynamik., Verschmutzung

Keywords : accidental pollution, city, system dynamics, water security

Mots-clés : dynamique des systèmes, pollution accidentelle, sécurité d'approvisionnement en eau, ville

\section{AUTEUR}

\section{CLAUDINE FERRON}

Laboratoire «Image et Ville » - UPRES-A 7011 CNRS - Université Louis-Pasteur - 3, rue de l'Argonne 67000 Strasbourg 